\title{
Research Article \\ Slant Curves in the Unit Tangent Bundles of Surfaces
}

\author{
Zhong Hua Hou and Lei Sun \\ Institute of Mathematics, Dalian University of Technology, Dalian, Liaoning 116024, China \\ Correspondence should be addressed to Lei Sun; mike.lei.sun@gmail.com
}

Received 26 September 2013; Accepted 25 October 2013

Academic Editors: T. Friedrich and M. Pontecorvo

Copyright (C) 2013 Z. H. Hou and L. Sun. This is an open access article distributed under the Creative Commons Attribution License, which permits unrestricted use, distribution, and reproduction in any medium, provided the original work is properly cited.

Let $(M, g)$ be a surface and let $(U(T M), G)$ be the unit tangent bundle of $M$ endowed with the Sasaki metric. We know that any curve $\Gamma(s)$ in $U(T M)$ consist of a curve $\gamma(s)$ in $M$ and as unit vector field $X(s)$ along $\gamma(s)$. In this paper we study the geometric properties $\gamma(s)$ and $X(s)$ satisfying when $\Gamma(s)$ is a slant geodesic.

\section{Introduction}

Let $(M, g, \phi, \xi, \eta)$ be a 3-dimensional contact metric manifold. The slant curves in $M$ are generalization of Legendrian curves which form a constant angle with the Reeb vector field $\xi$. Cho et al. [1] studied Lancret type problem for curves in Sasakian 3-manifold. They showed that a curve $\gamma(s) \subset M$ is slant if and only if $(\tau \pm 1) / k_{\gamma}$ is constant where $\tau$ and $k_{\gamma}$ are torsion and curvature of $\gamma(s)$, respectively, and they also gave some examples of slant curves. One can find some other papers about slant curves in almost contact metric manifolds. For examples, Călin et al. [2] studied the slant curves in $f$ Kenmotsu manifolds. In [3], Călin and Crasmareanu studied slant curves in normal almost contact manifolds.

Let $(M, g)$ be a Riemannian manifold. Sasaki $[4,5]$ studied the geometries of TM endowed with the Sasaki metric and introduced the almost complex structure $J$ in $T M$ which is compatible with $G_{s}$. Tashiro [6] constructed an almost contact metric structure $\left(G^{\prime}, \phi^{\prime}, \xi^{\prime}, \eta^{\prime}\right)$ in the unit tangent bundle $U(T M)$ of $M$ which is induced from the almost complex structure $\left(T M, G_{s}, J\right)$ in $T M$. Klingenberg and Sasaki [7] studied geodesics in the unit tangent bundle of 2-sphere $S^{2}$ endowed with Sasaki metric and showed that $U\left(T S^{2}\right)$ is isometric to $R P^{3}(1 / 4)$. Sasaki [8] studied the geodesics on the unit tangent bundles over space forms.

In this paper, we study the slant geodesics in the unit tangent bundle $U(T M)$ of some surface $M$. For any curve $\gamma(s)$ in $M$, let $T(s)=|T(s)| E(s)$ be the tangent vector field of $\gamma(s)$ and let $K_{M}(s)$ be the sectional curvature of $M$ at $\gamma(s)$, we have the following theorems.
Theorem 1. Let $\Gamma(s)=(\gamma(s), X(s))$ be a Legendrian geodesic parameterized by arc length in $U(T M)$ with domain $s \in[a, b]$. If the set consisting of points $s \in[a, b]$ such that $K_{M}(s)=1$ is discrete, then $\gamma(s)$ is a geodesic of velocity 2 and $X(s)$ is the normal direction of $\gamma$ in $M$.

Theorem 2. Let $\Gamma(s)=(\gamma(s), X(s))$ be a slant geodesic parameterized by arc length in $U(T M)$ which is not Legendrian. Under the assumptions of $K_{M}(s)$ as in Theorem 1, we have the following.

(1) If $X(s) \neq \pm E(s)$, then $\gamma(s)$ is a geodesic of velocity 2 and $X$ is a parallel vector field along $\gamma(s)$.

(2) If $X(s)= \pm E(s)$, then $\gamma(s)$ is a curve of velocity $2|\cos \theta|$ with constant curvature $k_{\gamma}= \pm \tan \theta$.

\section{Preliminaries}

Firstly, we introduce the (almost) contact metric structure on a Riemannian manifold of odd dimension. With the same notations as in [9]; let $M$ be a real $(2 n+1)$-dimensional $\mathscr{C}^{\infty}$ manifold and $\mathfrak{X}(M)$ the Lie algebra of $\mathscr{C}^{\infty}$ vector fields on $M$. An almost cocomplex structure on $M$ is defined by a $\mathscr{C}^{\infty}$ (1,1)-tensor $\phi$, a $\mathscr{C}^{\infty}$ vector field $\xi$ and a $\mathscr{C}^{\infty} 1$-form $\eta$ on $M$ such that for any point $x \in M$ we have

$$
\phi_{x}^{2}=-I+\eta_{x} \bigotimes \xi_{x}, \quad \eta_{x}\left(\xi_{x}\right)=1,
$$

where $I$ denotes the identity transformation of the tangent space $T_{x} M$ at $x$. Manifolds equipped with an almost 
cocomplex structure are called almost contact manifolds. A Riemannian manifold with a metric $g$ and an almost contact structure $(\phi, \xi, \eta)$ is called almost contact metric or almost coHermitian manifold if $g$ and $(\phi, \xi, \eta)$ satisfies

$$
g(\phi X, \phi Y)=g(X, Y)-\eta(X) \eta(Y),
$$

for any vector fields $X, Y \in \mathfrak{X}(M)$.

As in Kahler geometry, we can define the fundamental 2form $\Phi$ on the almost contact metric manifold $(M, g, \phi, \xi, \eta)$ as

$$
\Phi(X, Y)=g(X, \phi Y)
$$

for any vector fields $X, Y \in \mathfrak{X}(M)$. Obviously, this form satisfies $\eta \wedge \Phi^{n} \neq 0$ which means every almost contact metric manifold is orientable and that $(\eta, \Phi)$ defines an almost cosymplectic structure on $M$. If the fundamental 2-form $\Phi=$ $d \eta$, then we call $(M, g, \phi, \xi, \eta)$ a contact metric manifold.

Let $\left(x^{i}, u^{i}\right)$ be the locally coordinate systems on the tangent bundle $T M$ of $M$. Sasaki $[4,5]$ defined a metric $G_{s}$ on $T M$ which is the natural lifts of the metric $g$ on $M$ as follows:

$$
\begin{aligned}
& G_{s}\left(X^{h}, Y^{h}\right)=g(X, Y), \\
& G_{s}\left(X^{h}, Y^{v}\right)=0, \\
& G_{s}\left(X^{v}, Y^{v}\right)=g(X, Y),
\end{aligned}
$$

where $X, Y \in \mathfrak{X}(M)$ and $X^{h}=X^{i} \partial_{x^{i}}-X^{i} u^{j} \Gamma_{i j}^{k} \partial_{u^{k}}$ and $X^{v}=$ $X^{i} \partial_{u_{i}}$ are the horizontal and vertical lifts of $X$ at $(x, u)$ with respect to Levi-Civita connection $\nabla$ of $g$, respectively, and $\left\{\Gamma_{i j}^{k}\right\}$ are the Christoffel symbols of $\nabla$. The Levi-Civita connection $\bar{\nabla}$ of $G_{s}$ is defined as

$$
\begin{array}{ll}
\bar{\nabla}_{X^{h}} Y^{h}=\left(\nabla_{X} Y\right)^{h}-\frac{1}{2}\left(R_{X Y}^{M} u\right)^{v}, & \bar{\nabla}_{X^{v}} Y^{h}=\frac{1}{2}\left(R_{u X}^{M} Y\right)^{h}, \\
\bar{\nabla}_{X^{h}} Y^{v}=\left(\nabla_{X} Y\right)^{v}+\frac{1}{2}\left(R_{u Y}^{M} X\right)^{h}, & \bar{\nabla}_{X^{v}} Y^{v}=0,
\end{array}
$$

where $X, Y \in \mathfrak{X}(M)$ and $R^{M}$ is the curvature tensor on $(M, g)$. The almost complex structure $J$ on $T M$ which is compatible with $G_{s}$ is given by

$$
J X^{h}=X^{v}, \quad J X^{v}=-X^{h},
$$

for any vector field $X \in \mathfrak{X}(M)$.

We know that the normal vector field of the unit tangent bundle $U(T M)=\{(x, u) \in T M ; g(u, u)=1\}$ of $M$ at $(x, u) \in$ $U(T M)$ in $\left(T M, G_{s}\right)$ is $u^{v}$. Let $\mathfrak{X}(U(T M))=\left\{X^{h}+Y^{t} ; X, Y \in\right.$ $\mathfrak{X}(M)$ \} be the Lie algebra of $\mathscr{C}^{\infty}$ vector fields on $U(T M)$ where $X^{h}=X^{i} \partial_{x^{i}}-X^{i} u^{j} \Gamma_{i j}^{k}$ and $X^{t}=(X-g(X, u) u)^{v}$ are the horizontal and tangential lifts of $X$ at $(x, u) \in U(T M)$, respectively. Let $i: U(T M) \rightarrow T M$ be the including map from $U(T M)$ to $T M$ and this map induces a metric $G^{\prime}=i^{*} G_{s}$ in $U(T M)$ as follows:

$$
\begin{aligned}
& G^{\prime}\left(X^{h}, Y^{h}\right)=g(X, Y), \\
& G^{\prime}\left(X^{h}, Y^{t}\right)=0, \\
& G^{\prime}\left(X^{t}, Y^{t}\right)=g(X, Y)-g(X, u) g(Y, u),
\end{aligned}
$$

for any vector fields $X, Y \in \mathfrak{X}(M)$.

From Tashiro [6], we know that there is an almost contact metric structure $\left(G^{\prime}, \phi^{\prime}, \xi^{\prime}, \eta^{\prime}\right)$ in $U(T M)$ which is induced from the almost complex structure $J$ in TM such that

$$
J \circ i_{*}=i_{*} \circ \phi^{\prime}+\eta^{\prime} \bigotimes u^{v}, \quad J \circ i_{*}\left(\xi^{\prime}\right)=u^{v} .
$$

This implies that

$$
\eta^{\prime}\left(\xi^{\prime}\right)=1, \quad \phi^{\prime}\left(\xi^{\prime}\right)=0, \quad \eta^{\prime} \circ \phi^{\prime}=0 .
$$

From Blair et al. [10], at any point $(x, u) \in U(T M)$ we have

$$
\begin{gathered}
\xi^{\prime}=u^{h}, \\
\eta^{\prime}(\bar{X})=G^{\prime}\left(\bar{X}, u^{h}\right), \\
\Phi^{\prime}(\bar{X}, \bar{Y})=G^{\prime}\left(\bar{X}, \phi^{\prime} \bar{Y}\right)=2 d \eta^{\prime}(\bar{X}, \bar{Y}), \\
\phi^{\prime} X^{h}=X^{t}, \quad \phi^{\prime} X^{t}=-X^{h}+\eta^{\prime}\left(X^{h}\right) \xi^{\prime},
\end{gathered}
$$

where $X \in \mathfrak{X}(M)$ and $\bar{X}, \bar{Y} \in \mathfrak{X}(U(T M))$. Hence, we know that there is a contact metric structure $(G, \phi, \xi, \eta)$ in $U(T M)$ such that

$$
\eta=\frac{1}{2} \eta^{\prime}, \quad \xi=2 \xi^{\prime}, \quad \phi=\phi^{\prime}, \quad G=\frac{1}{4} G^{\prime} .
$$

By (5), we know that the Levi-Civita connection $D$ of $(U(T M), G)$ is determined by the following:

$$
\begin{aligned}
& D_{X^{h}} Y^{h}=\left(\bar{\nabla}_{X^{h}} Y^{h}\right)^{\top}=\left(\nabla_{X} Y\right)^{h}-\frac{1}{2}\left(R_{X Y} u\right)^{t}, \\
& D_{X^{t}} Y^{h}=\left(\bar{\nabla}_{X^{t}} Y^{h}\right)^{\top}=\frac{1}{2}\left(R_{u X} Y\right)^{h}, \\
& D_{X^{h}} Y^{t}=\left(\bar{\nabla}_{X^{h}} Y^{t}\right)^{\top}=\left(\nabla_{X} Y\right)^{t}+\frac{1}{2}\left(R_{u Y} X\right)^{h}, \\
& D_{X^{t}} Y^{t}=\left(\bar{\nabla}_{X^{t}} Y^{t}\right)^{\top}=-g(Y, u) X^{t},
\end{aligned}
$$

for any $(x, u) \in U(T M)$ and $X, Y \in \mathfrak{X}(U(T M))$.

\section{Geodesic Slant Curves in $U(T M)$}

Let $(M, g)$ be a surface and let $\gamma:[a, b] \rightarrow M$ be a curve in $M$. Let $\Gamma(s)=(\gamma(s), X(s))$ be a curve in $(U(T M), G, \phi, \xi, \eta)$, where the contact metric structure is given by (11).

Definition 3. We say that $\Gamma(s)$ is a slant curve in $U(T M)$ if the angle between the tangent vector field $\Gamma^{\prime}(s)$ of $\Gamma(s)$ and $\xi$ is constant. 
Assume that $\Gamma(s)$ is parameterized by the arc length. We know that

$$
\begin{aligned}
\Gamma^{\prime}(s) & =\frac{d x^{i}}{d s} \partial_{x^{i}}+\frac{d X^{i}}{d s} \partial_{u^{i}} \\
& =\frac{d x^{i}}{d s}\left(\partial_{x^{i}}\right)^{h}(\Gamma(s))+\left(\frac{d X^{i}}{d s}+\frac{d x^{j}}{d s} X^{k} \Gamma_{j k}^{i}\right) \partial_{u^{i}}(\Gamma(s)) \\
& =\left(T^{h}+\left(\nabla_{T} X\right)^{v}\right)(\Gamma(s)),
\end{aligned}
$$

where $T(s)=\gamma^{\prime}(s)$. Since $G\left(\Gamma^{\prime}, \Gamma^{\prime}\right)=1$ and $G=G^{\prime} / 4$ we have

$$
g(T, T)+g\left(\nabla_{T} X, \nabla_{T} X\right)=4 .
$$

Let $\theta$ be the angle between $\Gamma^{\prime}(s)$ and $\xi$. We have

$$
\cos \theta(s)=G\left(\Gamma^{\prime}(s), \xi\right)=\frac{g(T(s), X(s))}{2} .
$$

Taking the derivative on both sides of (15) with respect to $s$, we derive that

$$
\begin{aligned}
-\sin \theta \cdot \theta^{\prime}= & \frac{d}{d s} G\left(\Gamma^{\prime}(s), \xi\right) \\
= & G\left(D_{\Gamma^{\prime}(s)} \Gamma^{\prime}(s), \xi\right) \\
& +G\left(\Gamma^{\prime}(s), D_{\Gamma^{\prime}(s)} \xi\right) \\
= & k_{\Gamma} \eta\left(N_{\Gamma}\right)+G\left(\Gamma^{\prime}(s), 2\left(\nabla_{T} X\right)^{h}\right. \\
& \left.\quad+\left(R_{u \nabla_{T} X} u\right)^{h}-\left(R_{T u} u\right)^{t}\right) \\
= & k_{\Gamma} \eta\left(N_{\Gamma}\right)+8 g\left(\nabla_{T} X, T\right)-8 R\left(T, X, X, \nabla_{T} X\right),
\end{aligned}
$$

where $\theta^{\prime}=d \theta / d s, \xi(s)=2 X^{h}(s)$, and $k_{\Gamma}$ and $N_{\Gamma}$ are the curvature and the direction of the acceleration of $\Gamma(s)$, respectively. have

If $\Gamma(s)$ is a slant curve, that is, $\theta \equiv$ Const, from (16) we

$$
k_{\Gamma} \eta\left(N_{\Gamma}\right)+8 g\left(\nabla_{T} X, T\right)-8 R\left(T, X, X, \nabla_{T} X\right)=0 .
$$

Let $\{E, N\}$ be the Frenet frame on $\gamma(s)$. It follows from (15) that we have

$$
X=\frac{2 \cos \theta}{\alpha} E+2 \beta N
$$

where $\alpha=|T|$ and $\beta$ is a $\mathscr{C}^{\infty}$ function. It follows that

$$
\begin{aligned}
\nabla_{T} X & =\left(\frac{2 \cos \theta}{\alpha}\right)^{\prime} E+2 \cos \theta \cdot k_{\gamma} N+2 \beta^{\prime} N-2 \alpha \beta k_{\gamma} E \\
& =-2\left(\frac{\cos \theta}{\alpha^{2}} \alpha^{\prime}+\alpha \beta k_{\gamma}\right) E+2\left(k_{\gamma} \cos \theta+\beta^{\prime}\right) N .
\end{aligned}
$$

Substituting (19) into (14), we have

$$
\alpha^{2}+4\left(\frac{\cos \theta}{\alpha^{2}} \alpha^{\prime}+\alpha \beta k_{\gamma}\right)^{2}+4\left(k_{\gamma} \cos \theta+\beta^{\prime}\right)^{2}=4 .
$$

Since $g\left(X, \nabla_{T} X\right)=0$, from (15) and (19) we have

$$
T=2 \cos \theta X-\frac{2}{g\left(\nabla_{T} X, \nabla_{T} X\right)}\left(\frac{\cos \theta}{\alpha} \alpha^{\prime}+\alpha^{2} \beta k_{\gamma}\right) \nabla_{T} X .
$$

It follows that

$$
\begin{aligned}
R & \left(T, X, X, \nabla_{T} X\right) \\
& =-\frac{2\left((\cos \theta / \alpha) \alpha^{\prime}+\alpha^{2} \beta k_{\gamma}\right) R\left(\nabla_{T} X, X, X, \nabla_{T} X\right)}{g\left(\nabla_{T} X, \nabla_{T} X\right)} \\
& =-2\left(\frac{\cos \theta}{\alpha} \alpha^{\prime}+\alpha^{2} \beta k_{\gamma}\right) K_{M}(s) .
\end{aligned}
$$

It follows from (19) and (22) that (17) turns into

$$
k_{\Gamma} \eta\left(N_{\Gamma}\right)+2\left(\frac{\cos \theta}{\alpha} \alpha^{\prime}+\alpha^{2} \beta k_{\gamma}\right)\left(K_{M}-1\right)=0 .
$$
rem.

For the Legendrian curves, we have the following theo-

Theorem 1. Let $\Gamma(s)=(\gamma(s)$ and let $X(s))$ be a Legendrian geodesic parameterized by arc length in $(U(T M), G, \phi, \xi, \eta)$ with domain $s \in[a, b]$. Suppose that the set consisting of points $s \in[a, b]$ such that the sectional curvature of $M$ at $\gamma(s)$ satisfying $K_{M}(s)=1$ is discrete. Then $\gamma(s)$ is a geodesic of velocity 2 and $X(s)$ is the normal direction of $\gamma(s)$ in $M$.

Proof. Since $\Gamma(s)$ is a Legendrian geodesic, we have

$$
\cos \theta=0, \quad \beta= \pm \frac{1}{2}, \quad X= \pm N, \quad k_{\Gamma}=0 .
$$

Substituting these into (20) and (23), we have

$$
\begin{aligned}
& \alpha^{2}\left(1+k_{\gamma}^{2}\right)=4, \\
& \alpha^{2} k_{\gamma}\left(K_{M}-1\right)=0 .
\end{aligned}
$$

By the second equation of (25) and the assumption of $K(s)$ we have $k_{\gamma}=0$ which means $\gamma(s)$ is a geodesic in $M$. Substituting $k_{\gamma}=0$ into the first equation of (25) we have $\alpha=2$. This completes the proof of Theorem 1 .

For the non-Legendrian slant geodesics, we have the following theorem.

Theorem 2. Let $\Gamma(s)=(\gamma(s), X(s))$ be a slant geodesic in $U(T M)$ which is not Legendrian. Under the assumptions of $K_{M}$ as in Theorem 1, we have the following.

(1) If $X(s) \neq \pm E(s)$, then $\gamma(s)$ is a geodesic of velocity 2 and $X$ is a parallel vector field along $\gamma(s)$.

(2) If $X(s)= \pm E(s)$, then $\gamma(s)$ is a curve of velocity $2|\cos \theta|$ with constant curvature $k_{\gamma}= \pm \tan \theta$. 
Proof. By the assumption we know that $\theta \neq \pi / 2$, and form which and (18) we have $\beta \neq \pm 1 / 2$. Since $g(X, X)=1$, from (18) we have

$$
4\left(\frac{\cos \theta}{\alpha}\right)^{2}+4 \beta^{2}=1
$$

from which we derive that

$$
\frac{\cos \theta}{\alpha}= \begin{cases}\frac{\sqrt{1-4 \beta^{2}}}{2}, & \theta \in\left[0, \frac{\pi}{2}\right), \\ -\frac{\sqrt{1-4 \beta^{2}}}{2}, & \theta \in\left(\frac{\pi}{2}, \pi\right] .\end{cases}
$$

Suppose that $\theta \in[0, \pi / 2)$, then we have

$$
\alpha=\frac{2 \cos \theta}{\sqrt{1-4 \beta^{2}}} .
$$

Taking derivative on both side of this equation with respect to $s$, we have

$$
\frac{\cos \theta}{\alpha^{2}} \alpha^{\prime}=\frac{2 \beta \beta^{\prime}}{\sqrt{1-4 \beta^{2}}}
$$

It follows that

$$
\frac{\cos \theta}{\alpha} \alpha^{\prime}+\alpha^{2} \beta k_{\gamma}=\frac{4 \beta \cos \theta}{1-4 \beta^{2}}\left(\beta^{\prime}+k_{\gamma} \cos \theta\right) .
$$

Substituting (30) into (23), we have

$$
k_{\Gamma} \eta\left(N_{\Gamma}\right)+\frac{8 \beta \cos \theta}{1-4 \beta^{2}}\left(\beta^{\prime}+k_{\gamma} \cos \theta\right)\left(K_{M}-1\right)=0 .
$$

Remark 4. (31) also holds for $\theta \in(\pi / 2, \pi]$.

For proving Theorem 2, we need the following lemma.

Lemma 5. If there is some $s_{0} \in[a, b]$ such that $\beta\left(s_{0}\right)=0$, then $\beta$ is vanishing everywhere.

Proof. Substituting (30) into (20), we have

$$
\frac{1}{1-4 \beta^{2}}\left(\cos ^{2} \theta+\left(k_{\gamma} \cos \theta+\beta^{\prime}\right)^{2}\right)=1,
$$

and from which we derive that

$$
k_{\gamma} \cos \theta+\beta^{\prime}= \pm \sqrt{1-4 \beta^{2}-\cos ^{2} \theta}
$$

Since $k_{\Gamma}=0$ and under the assumption of $K(s)$, from (31) and (33) we have

$$
\beta\left(1-4 \beta^{2}-\cos ^{2} \theta\right)=0
$$

Let $I$ be a subset of $[a, b]$ such that $\left.\beta\right|_{I}=0$. Suppose that $I$ is not empty. From (34) we have

$$
\beta(s)=\frac{ \pm \sqrt{1-\cos ^{2} \theta}}{2},
$$

for any $s \in I-[a, b]$. It is a contradiction to the continuity of $\beta$ unless $\cos \theta= \pm 1$; that is, $\theta=0$ or $\pi$. Substituting $\theta=0$ or $\pi$ into (34) we have $\beta=0$ which means $I=[a, b]$. This completes the proof of the Lemma.

Firstly, we consider $I=\emptyset$ which implies $\beta \neq 0$. It follows from which and (18) we have $X \neq \pm E$.

From (34) we have

$$
\beta \equiv \frac{ \pm \sin \theta}{2}
$$

It follows from which and (27) we have $\alpha=2$. Substituting $\beta$ into (33) we have $k_{\gamma}=0$.

Substituting $\alpha, \beta$, and $k_{\gamma}$ into (19), we have $\nabla_{T} X=0$. This proves Case (1) in Theorem 2.

Secondly, we consider $I=[a, b]$ which implies $\beta \equiv 0$. It follows from which and (18) we have $X= \pm E$ and $\alpha=$ $2|\cos \theta|$.

Substituting $\alpha$ and $\beta$ into (33) we have

$$
k_{\gamma}= \pm \sqrt{\frac{1}{\cos ^{2} \theta}-1}= \pm \tan \theta
$$

which proves Case (2) in Theorem 2. This completes the proof of Theorem 2 .

\section{Acknowledgment}

This work was supported in part by the fundamental research funds for the Central University.

\section{References}

[1] J. T. Cho, J.-I. Inoguchi, and J.-E. Lee, "On slant curves in Sasakian 3-manifolds," Bulletin of the Australian Mathematical Society, vol. 74, no. 3, pp. 359-367, 2006.

[2] C. Călin, M. Crasmareanu, and M. I. Munteanu, "Slant curves in three-dimensional $f$-Kenmotsu manifolds," Journal of Mathematical Analysis and Applications, vol. 394, no. 1, pp. 400-407, 2012.

[3] C. Călin and M. Crasmareanu, "Slant curves in 3-dimensional normal almost contact geometry," Mediterranean Journal of Mathematics, vol. 10, no. 2, pp. 1067-1077, 2013.

[4] S. Sasaki, "On the differential geometry of tangent bundles of Riemannian manifolds," The Tohoku Mathematical Journal, vol. 10, pp. 338-354, 1958.

[5] S. Sasaki, "On the differential geometry of tangent bundles of Riemannian manifolds, II," The Tohoku Mathematical Journal, vol. 14, no. 2, pp. 146-155, 1962.

[6] Y. Tashiro, "On contact structure of hypersurfaces in complex manifolds. I," The Tohoku Mathematical Journal, vol. 15, pp. 6278, 1963.

[7] W. Klingenberg and S. Sasaki, "On the tangent sphere bundle of a $f$-sphere," The Tohoku Mathematical Journal, vol. 27, pp. 49$56,1975$. 
[8] S. Sasaki, "Geodesics on the tangent sphere bundles over space forms," Journal für die Reine und Angewandte Mathematik, vol. 288, pp. 106-120, 1976.

[9] D. Janssens and L. Vanhecke, "Almost contact structures and curvature tensors," Kodai Mathematical Journal, vol. 4, no. 1, pp. 1-27, 1981.

[10] D. E. Blair, Riemannian Geometry of Contact and Symplectic Manifolds, vol. 203 of Progress in Mathematics, Birkhäuser, Boston, Mass, USA, 2002. 


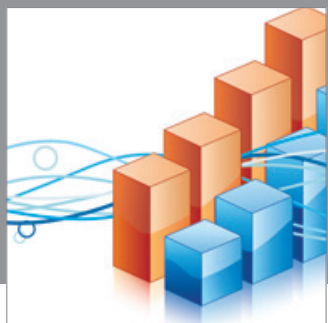

Advances in

Operations Research

mansans

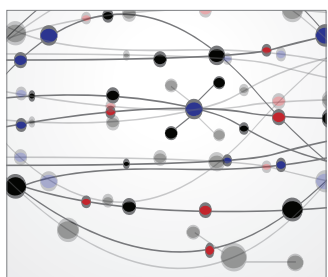

The Scientific World Journal
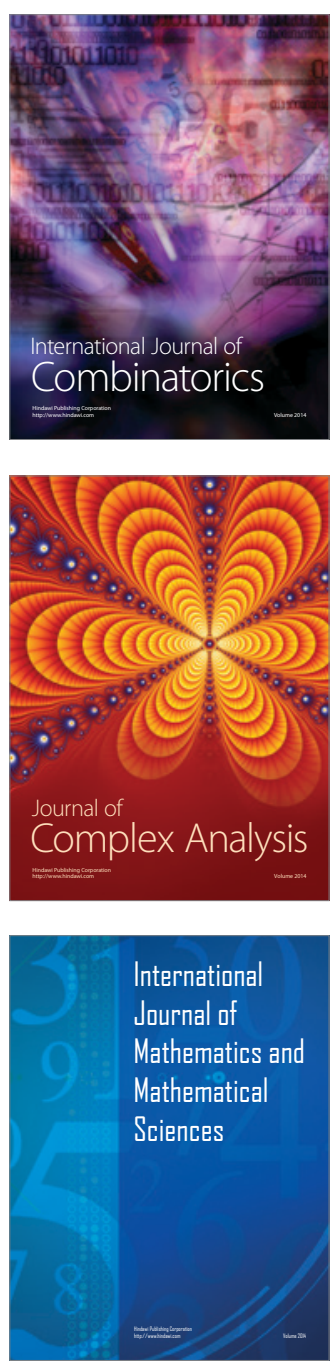
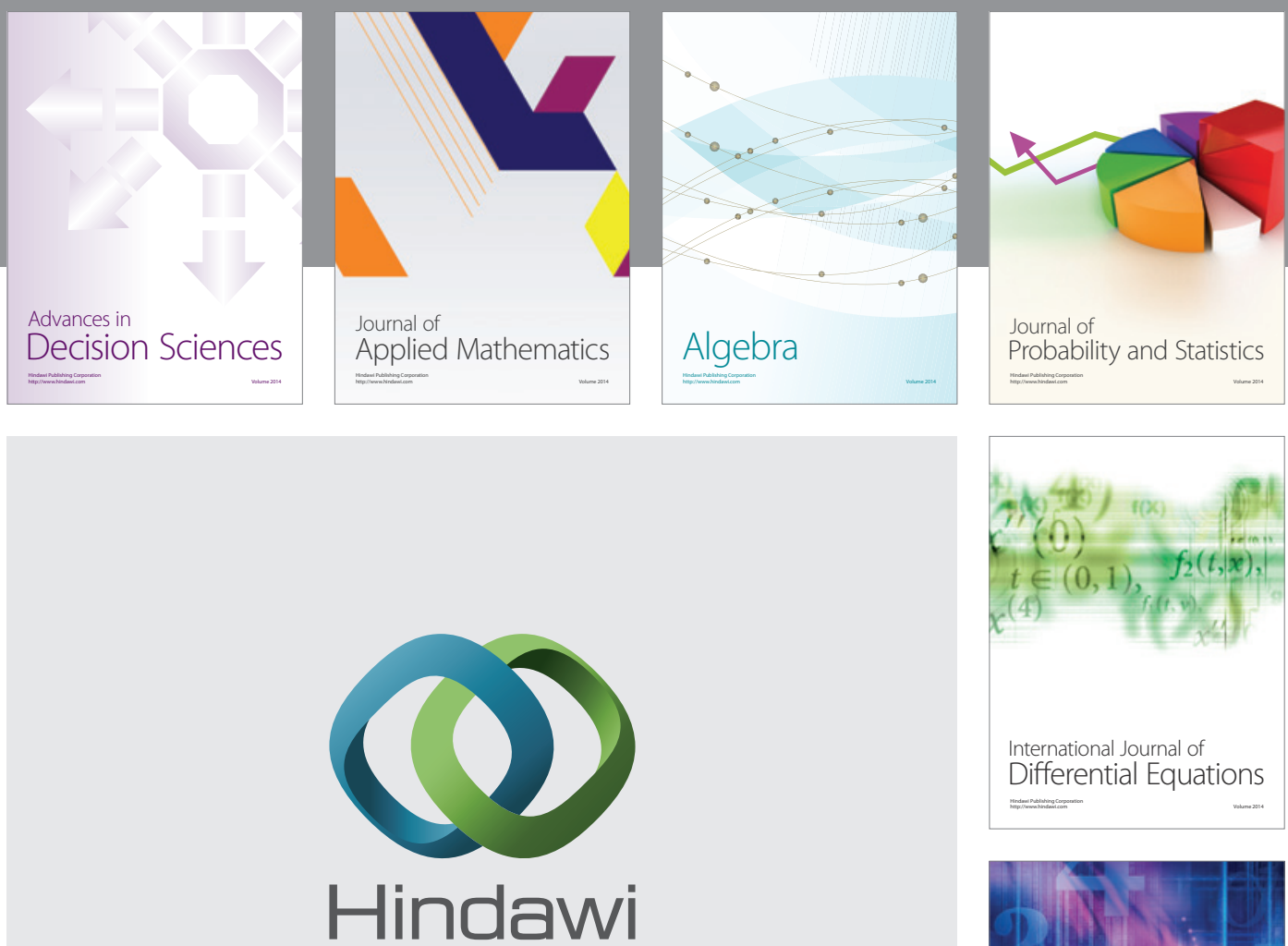

Submit your manuscripts at http://www.hindawi.com
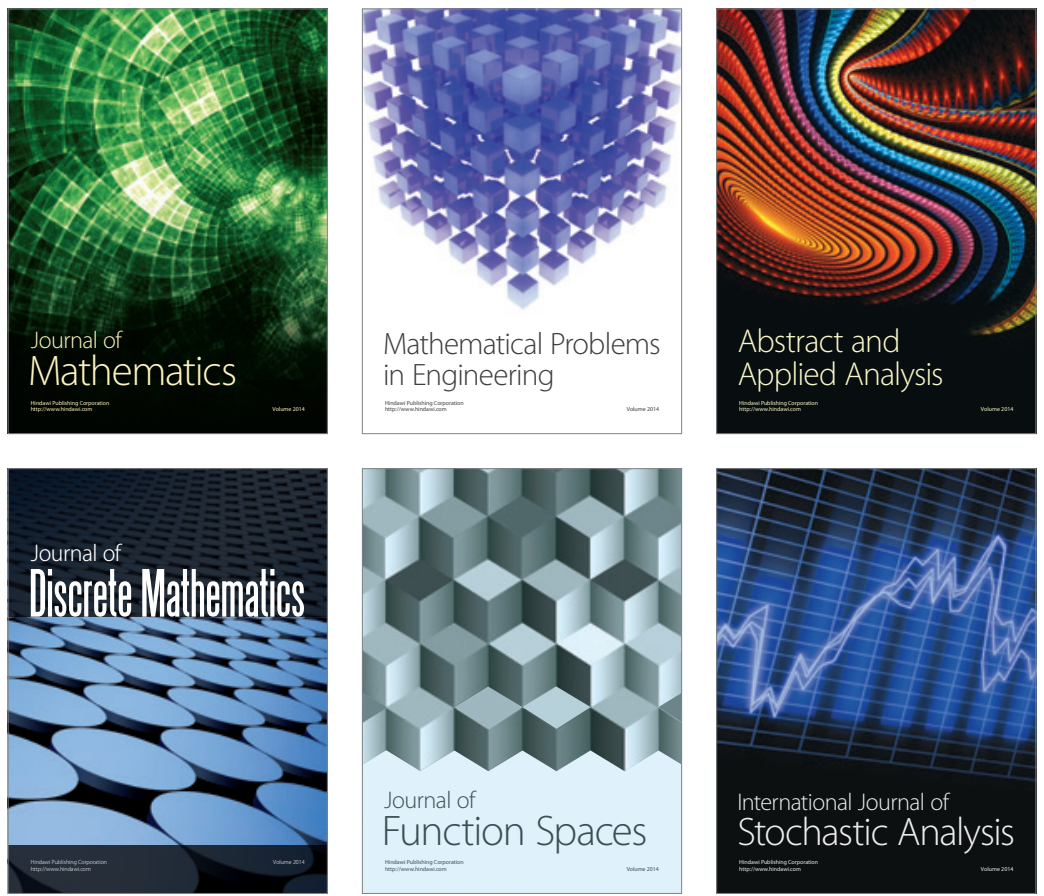

Journal of

Function Spaces

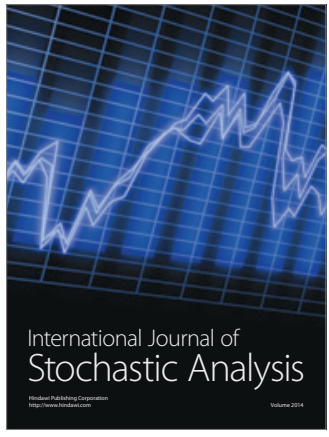

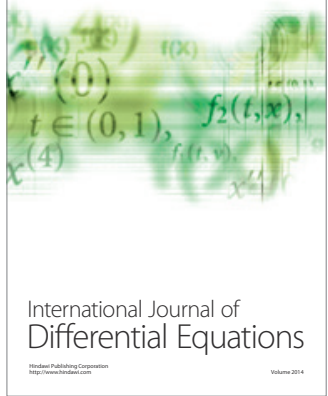
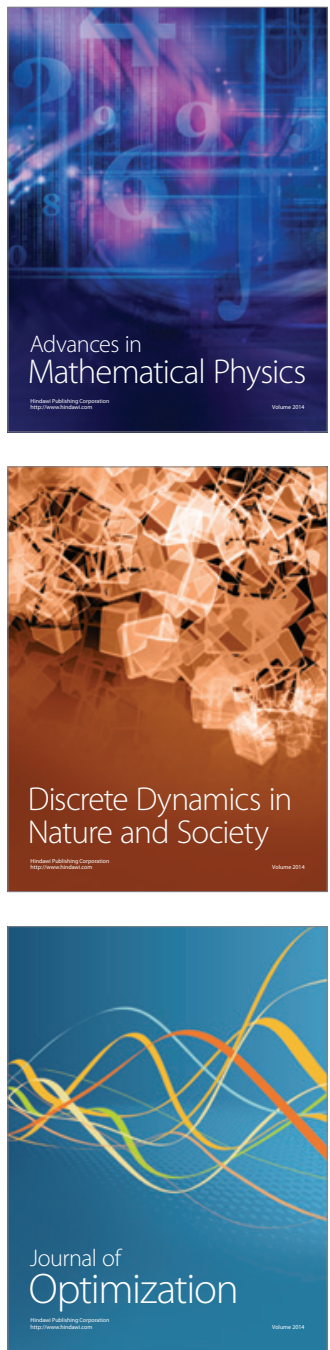